\title{
Propuesta de capacitación a directores de instituciones educativas del nivel preescolar basado en la crianza respetuosa en la nueva normalidad
}

\section{Training proposal for directors of educational institutions at the preschool level based on respectful parenting in the new normality}

Katelinen Mirian Rivera-Paipay

Universidad César Vallejo, Lima, Perú

katelinenriverapaipay09@gmail.com

https://orcid.org/0000-0003-3398-749X

María Elena Cornejo-Guevara

Universidad César Vallejo, Lima, Perú

ma.elena_2624@hotmail.com

(D) https://orcid.org/0000-0003-4235-941X

Carmen Ríos-Espinoza

Universidad César Vallejo, Lima, Perú

carmen_rios_9@hotmail.com

iD https://orcid.org/0000-0001-5274-7243

Magnet Liliane Vidal-Negreiros

Universidad César Vallejo, Lima, Perú

magnetvidal@hotmail.com

Recepción: 16/10/2021 | Aceptación: 06/01/2022 | Publicación: 10/01/2022

Cómo citar (APA, séptima edición):

Rivera-Paipay, K. M., Cornejo-Guevara, M. E., Ríos-Espinoza, C., y Vidal-Negreiros, M.L.

(2022). Propuesta de capacitación a directores de instituciones educativas del nivel preescolar basado en la crianza respetuosa en la nueva normalidad. INNOVA Research Journal, 7(1), 59-76. https://doi.org/10.33890/innova.v7.n1.2022.1971

\section{Resumen}

El presente estudio tuvo por objetivo identificar la influencia de un programa de capacitación para directivos de instituciones educativas de preescolar con actividades basadas en el enfoque de crianza respetuosa, haciendo uso de la estrategia de aprendizaje colectivo, para que de esta forma 
se genere un efecto multiplicador de conocimiento, hasta llegar a alcanzar logros en las actividades de los responsables del cuidado de los niños menores de seis años. La investigación es de enfoque cuantitativo, nivel explicativo, de diseño cuasiexperimental, el cual permitió acumular información relevante obtenida a través de la técnica de la observación, el instrumento cuenta con 16 ítems que posee una validez de contenido realizada a través de juicio de expertos y su confiabilidad estuvo determinada por un Alfa de Cronbach de 0.86. Es de corte transversal puesto que la información se recopiló durante los meses de marzo a junio del año 2021. La población estuvo conformada por 40 directores de instituciones educativas de nivel preescolar, de los cuales a 20 de ellos participaron en el programa. Los resultados obtenidos tras la aplicación de la prueba de U de Mann Whitney con un $\mathrm{p}=0,000$ evidenciaron que el programa de capacitación basado en el liderazgo directivo influyó en el conocimiento de la crianza respetuosa en la nueva normalidad.

Palabras claves: liderazgo directivo; crianza respetuosa; programa de capacitación; educación.

\begin{abstract}
The objective of this study was to identify the influence of a training program for managers of preschool educational institutions with activities based on the respectful parenting approach, making use of the collective learning strategy, so that in this way a multiplier effect is generated. of knowledge, until reaching achievements in the activities of those responsible for the care of children under six years of age. The research is of a quantitative approach, explanatory level, of quasi-experimental design, which allowed the accumulation of relevant information obtained through the observation technique, the instrument has 16 items that have a content validity made through the judgment of experts and its reliability was determined by a Cronbach's Alpha of 0.86 . It is cross-sectional since the information was collected during the months of March to June 2021. The population was made up of 40 directors of preschool educational institutions, of which 20 of them participated in the program. The results obtained after applying the Mann Whitney U test with a $\mathrm{p}=0.000$ showed that the training program based on directive leadership influenced the knowledge of respectful parenting in the new normal.
\end{abstract}

Keywords: executive leadership; respectful parenting; training program.

\title{
Introducción
}

Durante el año 2020, inició y se propagó una pandemia (Covid-19) a nivel global que obligó a distintas organizaciones e instituciones de diversos rubros a detener sus actividades, esta situación género que las personas realizaran un cambio vertiginoso en sus actividades cotidianas, adaptándolas a un entorno nuevo, en el cual no solo las personas que realizaban una actividad profesional o de oficio, necesitaban adecuarse a este cambio, sino también esta situación afectó a los niños y niñas en el hogar.

En el presente estudio se consideró realizar una propuesta de un programa el cual busca fortalecer los conocimientos de los directivos basado en el enfoque de la crianza respetuosa para que a partir de ello se pueda atender a las necesidades que han venido manifestando los padres de familia en las instituciones educativas en la cual se aplicó el programa. Para el desarrollo del estudio se realizó el análisis de diversas investigaciones en las cuales se logró contrastar que los resultados de las propuestas fueron eficaces en medida que los padres, directivos y docentes 
Propuesta de capacitación a directores de instituciones educativas del nivel preescolar basado en la crianza respetuosa en la nueva normalidad

participaron de manera activa con el propósito de buscar cambios positivos en los patrones conductuales de los infantes.

Cable News Network (CNN, 2020) publica en su sitio web, una columna referida a la educación, en la que se considera que a partir de la propagación del COVID-19, la educación asumirá desafíos importantes, dentro de los cuales se señala que la virtualidad los acogerá para que la educación no se detenga. Bustamante (2020) consideró que estamos pasando de una educación remota de emergencia a un retorno probable de modalidad semipresencial; mientras tanto Unicef Bolivia (2021) indica que en este contexto también existe la preocupación de cuál será el futuro que tendrá la educación de los niños y niñas, puesto que más allá de que el estado y las entidades afines a la educación estén realizando propuestas de retorno progresivo a las aulas, son los padres de familia los que están preocupados no solo por el tipo de calidad educativa que les será brindará a sus hijos sino también por la salud física y emocional de los mismos.

La Resolución Viceministerial $\mathrm{N}^{\circ}$ 097-2020-MINEDU promulgada por el Ministerio de Educación del Perú (2020) estableció una normativa con disposiciones sobre el trabajo remoto en instituciones que brindan servicio educativo; este documento brinda mecanismos de supervisión del trabajo en las cuales los directores desempeñan un rol protagónico. Considerando en el directivo habilidades como el liderazgo, responsabilidad, toma de decisiones, empatía, trabajo en equipo y compromiso social que deben reflejarse en su gestión.

Mena (2017) menciona que los directivos de instituciones educativas deben gestionar la escuela ejerciendo un liderazgo pedagógico; influyendo, inspirando y movilizando a la comunidad educativa hacia el cumplimiento de los objetivos educacionales. Estas condiciones abren puertas para que la triada de apoyo en la educación del niño, este más afianzada y consolidada. De este modo, surgen ideas como establecer programas en los que los gestores puedan retroalimentar, nutrir sus conocimientos y de esta forma generar aprendizajes colectivos; y que este a su vez sea replicado en una cadena que permita que cada uno de los actores de la comunidad educativa pueda conocer y entender alternativas de solución a problemáticas, como la crianza de los menores de edad en este contexto de pandemia.

Trabajar de la mano con los padres de familia y atender sus dudas es una de las principales labores educativas que se asumen en estos tiempos, estas orientaciones facilitarán un trabajo más encaminado a desarrollar los logros progresivos en sus niños. Sin embargo, es preciso un nuevo rumbo en las actividades parentales tratando de apostar por opciones que fortalecen positivamente nuestros vínculos familiares, visto de este modo la crianza respetuosa, se convierte en una forma de fortalecer la convivencia familiar que ha venido siendo afectada por la pandemia, tal como lo señala Ugaz, Oficial de Desarrollo Infantil Temprano de Unicef Perú (2021), "ha deteriorado el vínculo de apego entre las niñas y niños con sus padres, algo tan fundamental porque esta interacción genera un ambiente de confianza, seguridad y favorece un buen desarrollo".

Durante el inicio de la pandemia se observaron sucesos que reorganizaron el sistema educativo en instituciones de educación preescolar. Los directores como parte de su labor de gestión, identificaron diversas necesidades de su comunidad educativa, dentro de las cuales los docentes reportaron que los niños y niñas estaban presentando algunas dificultades para regular sus emociones, respetar las normas de conducta dentro del hogar, equilibrar el tiempo de juego y 
las actividades educativas; todo esto se contrastó con la realidad que se vive durante la pandemia pues el confinamiento hizo que las actividades laborales de los padres, los quehacer del hogar, las actividades educativas de los miembros de la familia se concentraron en un solo lugar y espacio. Los niños en su desarrollo no cuentan con la capacidad de adaptarse a estos cambios tan fácilmente, en consecuencia, los nuevos roles que asumieron los padres en sus hogares como orientadores educativos generó que se presentaran algunos déficits para establecer normas de conducta en el hogar; por lo que solicitaron el apoyo y orientación en sus respectivas instituciones educativas con la finalidad de hacer más llevadera la situación generada por esta nueva normalidad.

Frente a la problemática expuesta, esta investigación tuvo por objetivo identificar la influencia de un programa de capacitación basado en liderazgo directivo para comprobar si este influye significativamente en el conocimiento de la crianza respetuosa en la nueva normalidad.

\section{Marco Teórico}

González y Sáenz (2020) comentan que la crianza respetuosa es un paradigma que deriva de la intersección de dos puntos de vista uno es el "desarrollo humano" y el otro el de los "derechos humanos", con la finalidad de generar un nuevo concepto de niñez en el que se reconoce la condición ciudadana de los niños desde que nacen. Guevara et al. (2020) establecen en su investigación que si un niño recibe modelos agresivos, estrechamente relacionados a castigos continuos y además de ello convive en un ambiente donde no se caracteriza la interacción social positiva, manifestara cambios conductuales que no le permitirán relacionarse; además, establecen que es importante fomentar desde el hogar la retroalimentación, reforzamiento y valores en los primeros años; por ello, es importante considerar que dirigiendo la crianza de los niños bajo opciones vinculadas al respeto, se podrán establecer logros importantes para su futura convivencia respetuosa con los demás miembros de su entorno (p.4). Di Stefano (2020) indica que esta propuesta de una crianza respetuosa apela a una metáfora naturalista que busca construir experiencias más íntimas, 'instintivas' y vivenciales entre padres e hijos. Es por ello, que propiciaría un desarrollo en el cual se ve fortalecido el lado humano de los menores.

A partir de la conceptualización de este nuevo paradigma de la crianza respetuosa, se puede apreciar que los niños son el eje central de su desarrollo; por lo cual, participan activamente en su proceso los padres o cuidadores, encaminados bajo los lineamientos de la crianza respetuosa con lo cual serán capaces de acompañarlos en actividades como el reflexionar, conocer las causas y consecuencias de sus acciones; y a su vez el de las otras personas, con el fin de que adquieran progresivamente elementos para lograr su autorregulación. De esta forma, se entiende que, primero se necesita tener la confianza de ser una persona valiosa, reconocida y escuchada para posteriormente ir autorregulando las acciones propias en un entorno social. (González y Sáenz, 2020)

La crianza "respetuosa", también denominada crianza "natural" o "fisiológica", ha adquirido mayor relevancia en las últimas décadas, países como Argentina, Chile y México vienen realizando propuestas en las cuales se favorezcan el buen trato y el respeto de los derechos de cada uno de los niños, estableciendo incluso en sus políticas educativas lineamientos importantes para que los actores educativos intervengan y conozcan los beneficios de su aplicación. La crianza respetuosa, plantea una forma de relacionarse con los niños donde se coloca la satisfacción de ese 
Propuesta de capacitación a directores de instituciones educativas del nivel preescolar basado en la crianza respetuosa en la nueva normalidad

encuentro en primer plano. Los discursos sobre crianza respetuosa enfatizan la búsqueda de la transformación personal que supone el ejercicio de este estilo parental que llevan adelante este tipo de crianza, que además concibe la maternidad y la paternidad como una oportunidad de aprendizaje, crecimiento personal y transformación espiritual (Mantilla, 2019).

De acuerdo con Di Stefano (2020) la "crianza con apego", "crianza natural" o "crianza respetuosa", surge en determinados grupos medios urbanos como una propuesta novedosa de socialización infantil, logrando crear un critica a los métodos tradicionales de crianza, en especial al biomédico referido a la pediatría, el cual indica formas estandarizadas para la crianza y cuidado de los menores. Por lo cual, esta propuesta hace alusión a una metáfora naturalista que busca construir experiencias más íntimas y vivenciales entre padres e hijos.

Según el Instituto Colombiano de Bienestar Familiar (2015) la crianza con respeto, esta enfoca en promover una educación basada en normas y estrategias que impartan disciplina con amor, logrando una socialización entre los niños y niñas mediante el reconocimiento y afecto; sin ejercer algún tipo de violencia sobre los mismos. Debido a esto la crianza con respeto cumple un papel integrador al permitir reflexionar el rol que cumplen los padres o cuidadores, al ser la primera línea de influencia de los niños y niñas. Mamauag et al. (2021) realizaron un estudio con familias filipinas en las cuales se trabajó una propuesta basada en el dialogo y la reflexión de sus miembros para direccionar las conductas inapropiadas que podían manifestar sus niños durante el desarrollo, las familias de menores recursos presentaban mayor indisposición para cambiar sus patrones de conducta sin embargo bajo orientación y retroalimentación se logró fortalecer la interacción verbal para que se fomentaran practicas positivas basadas en el respeto. Instituciones e investigadores vienen demostrando a través de sus estudios que se obtienen resultados efectivos si se trabaja la crianza a través del fomento de valores y respeto.

Duarte (2019) realizó un plan de intervención en el cual se involucraba la atención a una dificultad familiar y pudo concluir que siguiendo un acompañamiento en el que las pautas de crianza respetuosa pueden direccionar cada uno de los roles que deben llevar a cabo cada miembro de la familia, será importante, y permitir cumplimiento de las normas en el hogar. Es fundamental que la familia sea quien debe orientarse sobre una educación adecuada para poder guiar e inculcar los valores que ayuden al crecimiento personal y bienestar del individuo. A través de las experiencias positivas que tenga el sujeto va permitiendo una relación positiva entre cada uno de los miembros de la familia.

Asimismo, Paliwoda (2020) refiere que, dentro de la atención a la primera infancia, la correcta manera de establecer límites en los infantes donde no se ejerza la violencia, favorece a futuro el desarrollo de intervenciones asertivas ante situaciones sociales. Por tanto, es necesario la presencia de un ambiente que permita trasmitir orientaciones que formen y preparen a los niños para que se relacionen apropiadamente respetándose y respetando a los demás.

Por otro lado, los especialistas del Ministerio de Educación de Perú (2021) señalan que los talleres para padres de familia en la educación básica regular permiten que desarrollen habilidades y estrategias para una crianza respetuosa donde se considera principalmente y ante todo el buen trato; y la comunicación con los menores (p. 68). De lo anteriormente señalado, podemos encontrar el punto de intersección entre la parte educativa y los padres de familia, en relación a que, parte de 
una crianza respetuosa se encuentra también asociada a como las instituciones educativas pueden guiar o instruir a los padres en esta temática.

Nativ et al. (2020) sostienen que la crianza respetuosa, no busca construir una técnica o normativa de crianza; sino trata de mostrar a los padres y cuidadores de una manera simple, que los niños son también personas que cuentan con derechos en la sociedad, los cuales deben desarrollarse en contextos donde se facilite su bienestar, tanto físico como emocional, a través del buen trato, evitando en lo posible exponerlos a factores de riesgo que interfieran en su desarrollo. Por ende, esta perspectiva de crianza no busca obtener algún fin o resultado en particular, sino que está más orientada a la salud emocional de los menores.

En estos dos últimos años, la educación se ha visto situada en un nuevo contexto, en el que si bien es cierto se han venido realizando actividades para evitar que se limite el aprendizaje, se han logrado generar nuevos espacios de trabajo, planificación y coordinación en los que han participado docentes, padres de familia y directivos; todos en búsqueda de estrategias que les permitan sobrellevar el día a día de esta nueva realidad. Dentro de una cultura institucional es importante identificar a las autoridades que son los principales gestores de las ideas y propuestas, en vista de tantas dificultades que se dieron durante esta nueva normalidad, los directores de las instituciones educativas han sido una pieza elemental para que se pueda establecer una adecuada detección de las necesidades, no fue novedoso escuchar que la conducta de los niños con el pasar de las semanas desde el aislamiento fue cambiando y esto origino que las familias soliciten orientación; pues al hablar también de una crianza respetuosa y como esta permite tener una interacción positiva, es prescindible contar con profesionales que practiquen y conozcan este tipo de enfoque.

Tagle (2017) considera que debe establecerse como una de las funciones del director, guiar y orientar al colectivo al logro de los propósitos de la institución, considerando el sistema básico de mejora (p.1). En las instituciones educativas de nivel inicial se presentó una realidad que tuvo que ser asistida casi de forma inmediata, los padres de familia pasaron a formar parte de las clases virtuales y ser el apoyo escolar en sus hogares, utilizando diversas estrategias para poder dar el soporte y trabajar con sus niños, estas situaciones fueron recogidas como testimonio por parte de muchos docentes y trasladada hacia sus directivos para generar alternativas de solución. Las mesas de trabajo en las instituciones de educación inicial manifestaban realizar acciones; es así que el liderazgo directivo toma el protagonismo, al generar alternativas que permitan fortalecer el conocimiento de los docentes respecto a este nuevo enfoque y como debe ser aplicando, a través de estrategias de gestión que permitan guiar el acompañamiento y monitoreo de los menores en sus hogares; y permitan el trasladado de este nuevo enfoque siguiendo un flujo de transmisión de conocimiento de directivos a docentes y de docentes hacia padres de familia.

Galiya et al. (2018) consideraron que es posible garantizar la calidad educativa y la atención a las necesidades de los niños, si los gestores y/o responsables de las instituciones educativas, evidencian competencias como el liderazgo, la gestión de habilidades y planes de acción inmediata para gestionar cambios; esta última es sobre todo una de las más relevantes, pues es un canal que permite brindar alternativas frente a situaciones atípicas e inusuales que propicien resultados para mejorar la práctica profesional del equipo que lidera; lo mencionado, permite entender que un directivo debe estar en la capacidad de buscar alternativas, propuestas, fortalecer 
sus conocimientos e incluso abrir puertas a nuevos enfoques que le permitan dar atención inmediata a problemas que puedan surgir durante su gestión. Tagle (2017) menciona que el liderazgo es una dimensión de la gestión educativa estratégica, el cual permite asumir que los retos a emprender necesitan ser identificados y planteados como problemas colectivos, evaluados en función de estos colectivos y de los valores en los que se van a profundizar para tener resultados positivos en la sociedad (p.4).

Rueda y Velásquez (2020) indicó que el liderazgo directivo está orientado a distribuir y fomentar el liderazgo entre los colaboradores de la institución educativa, teniendo en cuenta el incentivar la motivación propia y trascedente de los mismos (p.32). Un director de institución educativa preescolar debe realizar prácticas apropiadas en bien de la comunidad escolar; el compromiso que asuma para resolver problemáticas tan urgentes como direccionar prácticas de crianza basada en el afecto evidenciara una cualidad alineada a gestionar la enseñanza aprendizaje en la escuela. Leithwood y Riehl (2005, como se citó en Anderson, 2010) consideran que un liderazgo directivo se puede identificar a través de prácticas clave como la dirección a futuro, el desarrollar personas, rediseñar la organización y gestionar la instrucción. El directivo de educación preescolar, debe de mostrar su destreza para desarrollar las capacidades de sus aliados educativos, docentes y familias; preparándolos en un enfoque tan humano y necesario para la vida futura de los niños y niñas. asimismo, Vallejo y Torres (2020) indican que es importante identificar que el personal docente de educación infantil se encuentre involucrado y comprometido en el trabajo activo con las familias, pues en este nuevo contexto es un factor importante para el desarrollo del proceso de enseñanza - aprendizaje y sobre todo el inculcar valores importantes para la vida, como es el respeto entre las personas.

Tomando como referencia las características de liderazgo directivo de Leithwood y Riehl (2005 como se citó en Anderson, 2010) y la temática sobre crianza cotidiana definida por Durrant (2013) que consiste en identificar y mantener los objetivos y metas, proporcionar calidez adecuada y soporte, comprender a la niñez desde sus pensamientos y sentimientos y concentrarse en la solución de los problemas; se procedió a estructurar un programa basado en la crianza respetuosa el cual contienen 16 sesiones y fue aplicado a los directivos de las instituciones educativas de educación inicial, el cual se muestra a continuación: 


\section{Figura 1}

\section{Estructura del Programa basado en la crianza respetuosa}
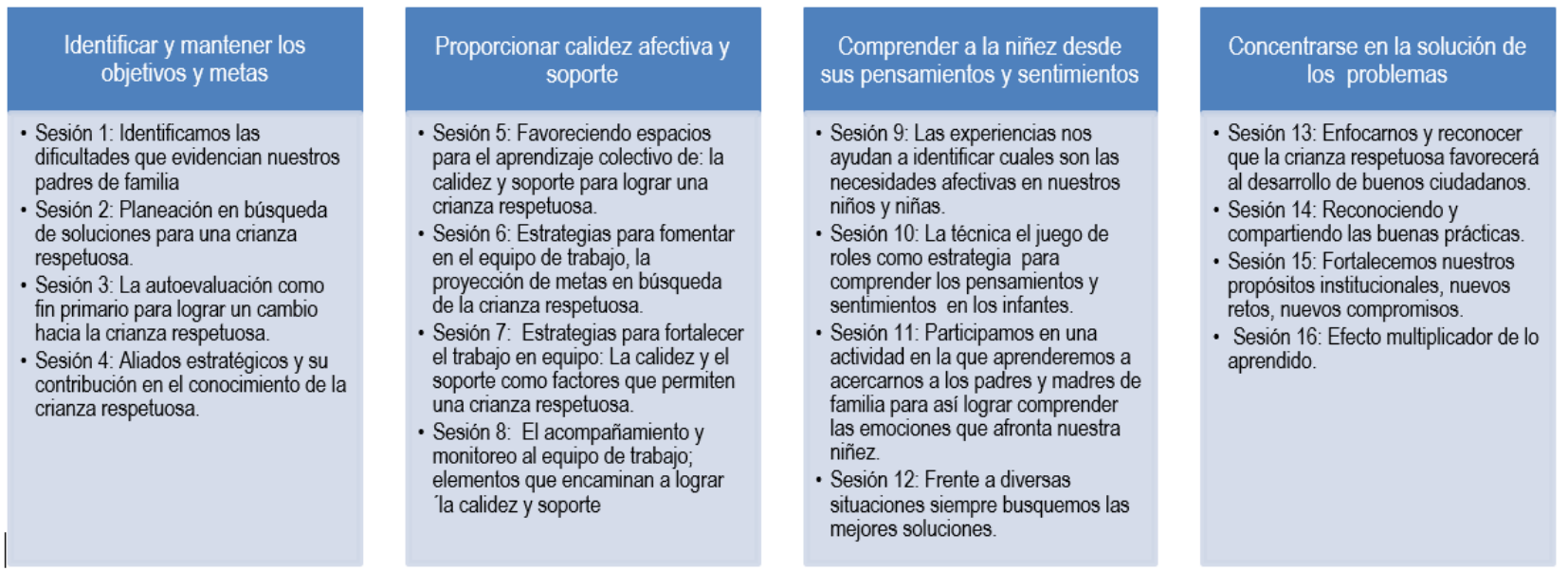

Nota. Programa basado en la crianza respetuosa, dividido en 4 módulos y 16 sesiones.

\section{Metodología}

La investigación fue realizada a través del enfoque cuantitativo el cual a través de la recolección de datos y el análisis estadístico permite probar la hipótesis planteada en la presente investigación (Hernández et al., 2014). Es de tipo es aplicada y cuenta con un diseño cuasiexperimental.

$\begin{array}{lll}\mathrm{GE}: \mathrm{O}_{1} & \mathrm{X} & \mathrm{O}_{2} \\ \mathrm{GC}: \mathrm{O}_{1} & - & \mathrm{O}_{2}\end{array}$

Donde:

GE: Representa al grupo experimental

GC: Representa al grupo control

$\mathrm{X}$ : Aplicación del programa

$\mathrm{O}_{1}$ : Pre- test

$\mathrm{O}_{2}$ : Post- test

La muestra estuvo conformada por 40 directores de instituciones educativas de educación básica regular del nivel inicial de diversos distritos de Lima Metropolitana, el tamaño de la muestra fue seleccionada mediante el muestreo de tipo no probabilístico por conveniencia, debido a que se tomará de acuerdo con el tipo y nivel de investigación, así como a la conveniencia del investigador, se autoselecciona en base a su disponibilidad y características que beneficien al estudio. (Hernández et al., 2014), siendo la distribución de: 20 directores para el grupo control y 20 directores para el grupo experimental a quienes se les aplicó un pretest y un postest. 
Propuesta de capacitación a directores de instituciones educativas del nivel preescolar basado en la crianza respetuosa en la nueva normalidad

Se realizó un registro de observación, el cual fue sometido a validez y confiabilidad, lográndose determinar en los resultados la viabilidad de su aplicación. El registro cuenta con un total de 16 ítems y un formato de respuestas politómicas.

\section{Tabla 1}

Distribución de las preguntas del registro de observación crianza respetuosa

\begin{tabular}{llc}
\hline Variable & Dimensión & Distribución de preguntas \\
\hline & $\begin{array}{l}\text { Identificar y mantener los objetivos y } \\
\text { metas. }\end{array}$ & $1,2,3$ y 4 \\
Crianza respetuosa & $\begin{array}{l}\text { Proporcionar calidez afectiva y soporte. } \\
\text { Comprender a la niñez desde sus } \\
\text { pensamientos y sentimientos. } \\
\begin{array}{l}\text { Concentrarse en la solución de los } \\
\text { problemas. }\end{array}\end{array}$ & $5,6,7$ y 8 \\
\hline
\end{tabular}

Nota: Elaboración propia

\section{Análisis de la información}

\section{Figura 2}

Niveles de distribución de la crianza respetuosa pretest y postest

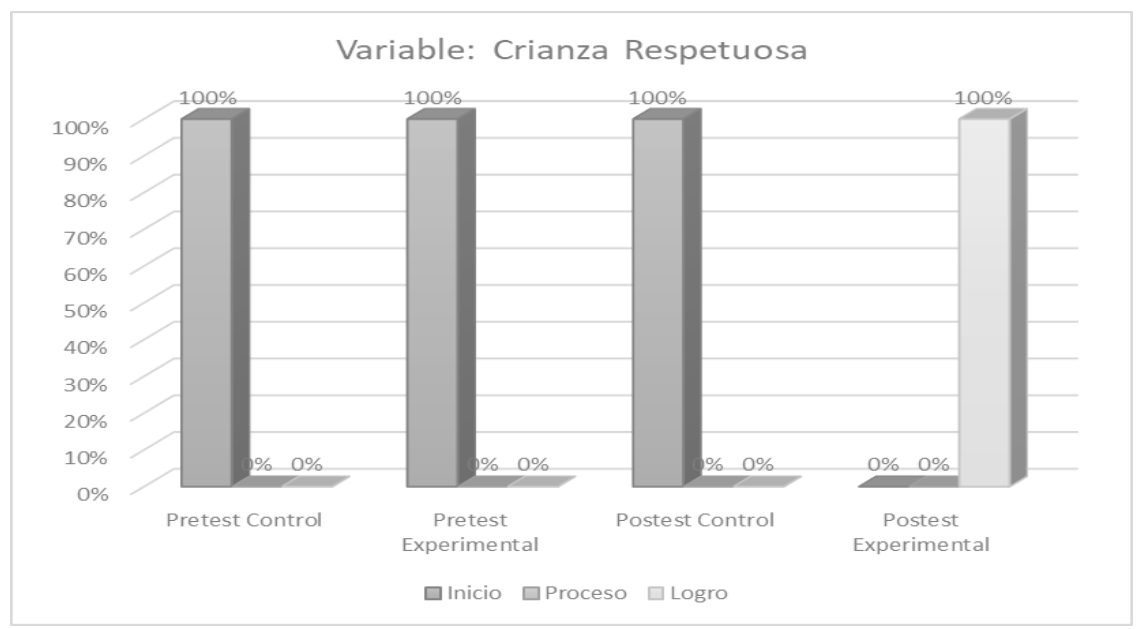

Nota: Base de datos

En figura 2, en cuanto a su Conocimiento sobre la crianza respetuosa, se evidencia que, en el pretest el $100 \%$ del grupo control y el $100 \%$ del grupo experimental se encuentran en un nivel de inicio. En cuanto al postest se observa que el 100\% del grupo control se encuentra en el nivel de inicio mientras que del grupo experimental el $100 \%$ tiene un nivel logrado. 


\section{Figura 3}

Niveles de distribución de la dimensión Identificar y mantener los objetivos y metas pretest y postest

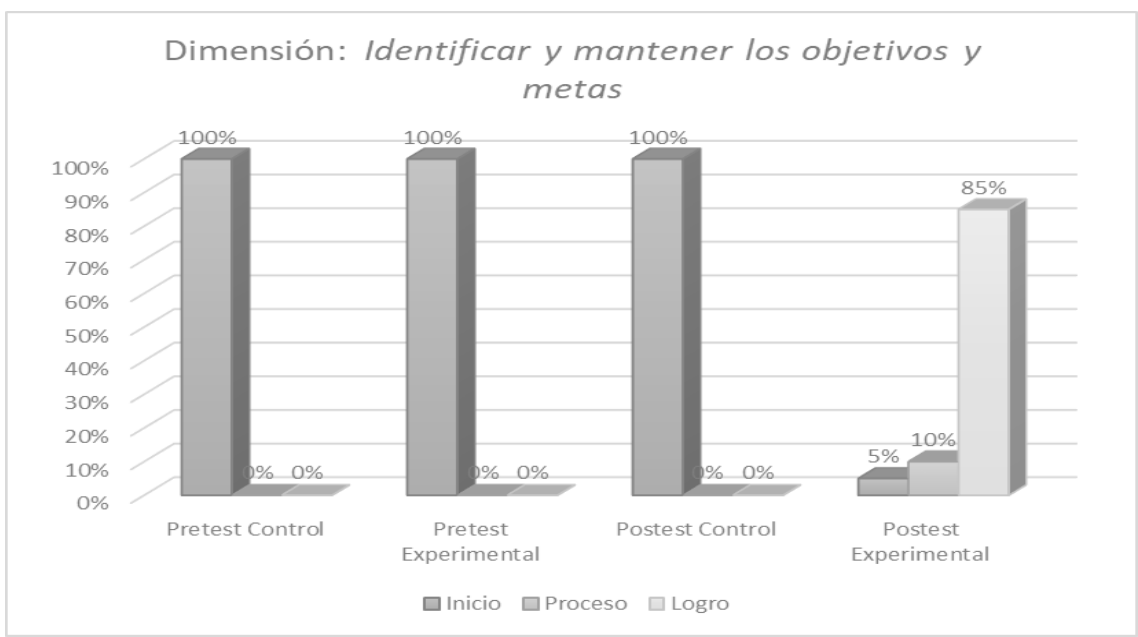

Nota: Base de datos

En figura 3, correspondiente a la dimensión Identificar y mantener los objetivos y metas, se evidencia que, en el pretest el 100\% del grupo control y el 100\% del grupo experimental se encuentran en un nivel de inicio, En cuanto al postest se observa que el 100\% del grupo control se encuentra en el nivel de inicio; mientras que el grupo experimental el 5\% tiene un nivel de inicio, el $10 \%$ se encuentra en el nivel de proceso y el $85 \%$ estén el nivel logrado.

\section{Figura 4}

Niveles de distribución de la dimensión Proporcionar calidez afectiva y soporte pretest y postest

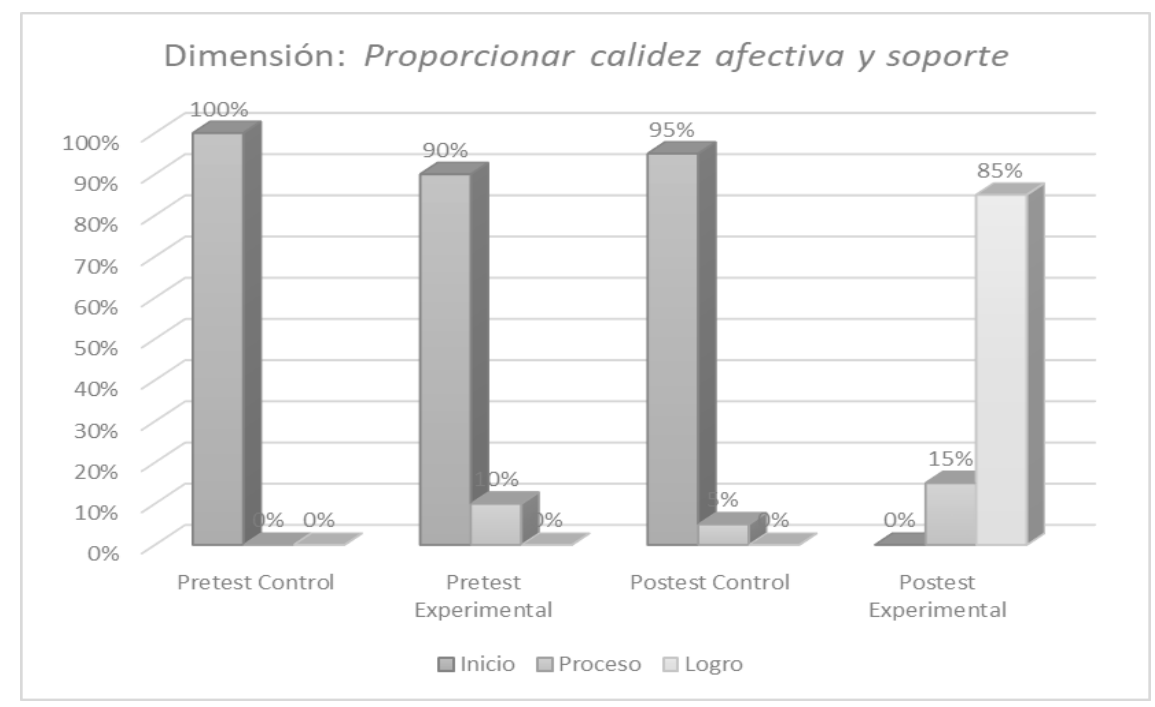


Nota: Base de datos

En la figura 4, correspondiente a la dimensión Proporcionar calidez afectiva y soporte, se evidencia que, en el pretest el 100\% del grupo control está en el nivel de inicio, mientras que en el grupo experimental el $90 \%$ está en el nivel de inicio y el 10\% está en el nivel proceso. En el postest se observa que el $95 \%$ del grupo control se encuentra en el nivel de inicio y el 5\% se encuentra en proceso; mientras que en el grupo experimental el $15 \%$ se encuentra en el nivel de proceso y el $85 \%$ se encuentra en el nivel de logro.

\section{Figura 5}

Niveles de distribución de la dimensión Comprender a la niñez desde sus pensamientos y sentimientos pretest y postest

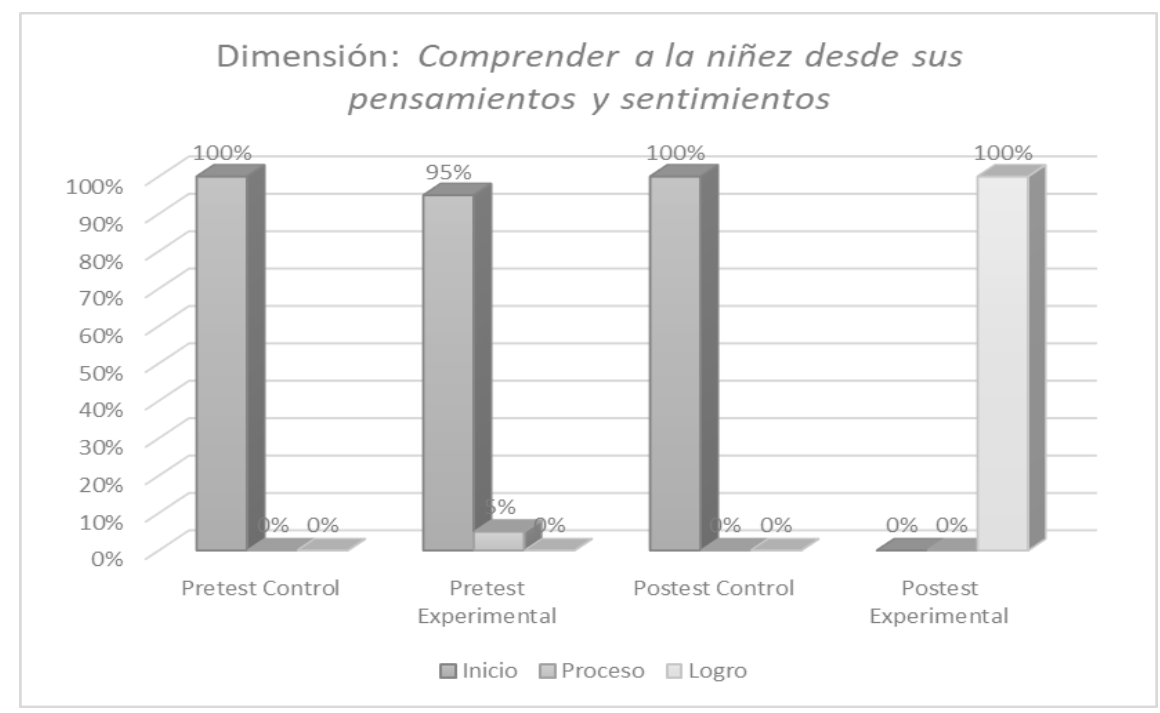

Nota: Base de datos

En la figura 5, correspondiente a la dimensión Comprender a la niñez desde sus pensamientos y sentimientos, se evidencia que, en el pretest el 100\% del grupo control está en inicio, mientras que en el grupo experimental el 95\% está en el nivel de inicio y el 5\% está en el nivel de proceso. En el postest se observa que el 100\% del grupo control se encuentra en inicio; mientras que en el grupo experimental el $100 \%$ se encuentra en el nivel logrado. 


\section{Figura 6}

Niveles de distribución de la dimensión Concentrarse en la solución de los problemas pretest y postest

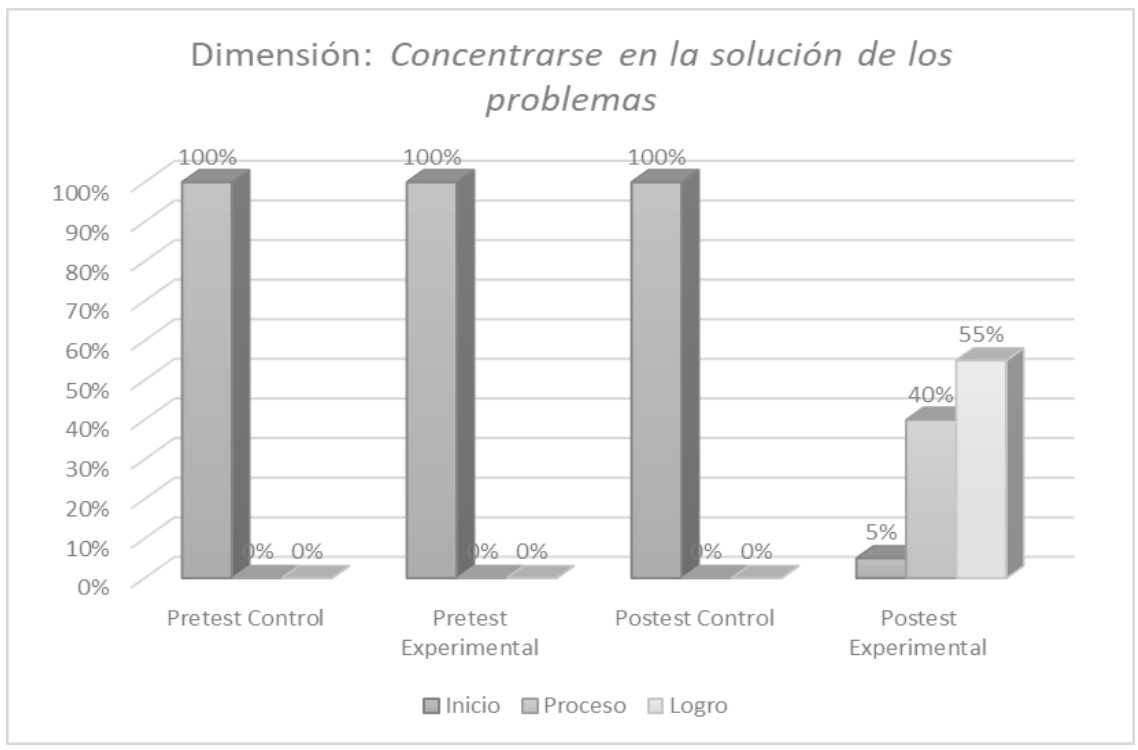

Nota: Base de datos

En la figura 6, correspondiente a la dimensión Concentrarse en la solución de los problemas, se observa que, en el pretest el 100\% del grupo control está en inicio y el 100\% del grupo experimental está en el nivel de inicio. En el postest se observa que el $100 \%$ del grupo control se encuentra en inicio; mientras que en el grupo experimental el 5\% se encuentra el nivel inicio, el $40 \%$ está en el nivel de proceso y el $55 \%$ en el nivel logrado.

\section{Tabla 2}

Prueba de normalidad

\begin{tabular}{|c|c|c|c|c|c|c|}
\hline & \multicolumn{3}{|c|}{ Kolmogorov-Smirnov ${ }^{\mathrm{a}}$} & \multicolumn{3}{|c|}{ Shapiro-Wilk } \\
\hline & Estadístico & gl & Sig. & Estadístico & gl & Sig. \\
\hline Crianza respetuosa pretest & ,200 & 40 &, 000 &, 868 & 40 &, 000 \\
\hline $\begin{array}{l}\text { Identificar y mantener los } \\
\text { objetivos y metas pretest }\end{array}$ & ,403 & 40 &, 000 & 614 & 40 & 000 \\
\hline $\begin{array}{l}\text { Proporcionar calidez afectiva y } \\
\text { soporte pretest }\end{array}$ & ,407 & 40 &, 000 &, 595 & 40 & 000 \\
\hline $\begin{array}{l}\text { Comprender a la niñez desde sus } \\
\text { pensamientos y sentimientos } \\
\text { pretest }\end{array}$ & 399 & 40 &, 000 &, 578 & 40 & 000 \\
\hline $\begin{array}{l}\text { Concentrarse en la solución de los } \\
\text { problemas pretest }\end{array}$ & 457 & 40 &, 000 &, 573 & 40 & 000 \\
\hline Crianza respetuosa postest &, 252 & 40 &, 000 & ,765 & 40 &, 000 \\
\hline $\begin{array}{l}\text { Identificar y mantener los } \\
\text { objetivos y metas postest }\end{array}$ & 239 & 40 &, 000 & ,783 & 40 & 000 \\
\hline $\begin{array}{l}\text { Proporcionar calidez afectiva y } \\
\text { soporte postest }\end{array}$ &, 230 & 40 &, 000 & ,816 & 40 & 000 \\
\hline
\end{tabular}


Propuesta de capacitación a directores de instituciones educativas del nivel preescolar basado en la crianza respetuosa en la nueva normalidad

\begin{tabular}{|c|c|c|c|c|c|c|}
\hline & \multicolumn{3}{|c|}{ Kolmogorov-Smirnova } & \multicolumn{3}{|c|}{ Shapiro-Wilk } \\
\hline & Estadístico & $\mathrm{gl}$ & Sig. & Estadístico & $\mathrm{gl}$ & Sig. \\
\hline $\begin{array}{l}\text { Comprender a la niñez desde sus } \\
\text { pensamientos y sentimientos } \\
\text { postest }\end{array}$ & ,238 & 40 &, 000 &, 754 & 40 &, 000 \\
\hline $\begin{array}{l}\text { Concentrarse en la solución de los } \\
\text { problemas postest }\end{array}$ & ,242 & 40 &, 000 & ,802 & 40 &, 000 \\
\hline
\end{tabular}

Nota: Base de datos.

En la tabla 2 se observa que en las dimensiones el valor de la significancia es $0,00<0,05$ para la prueba de normalidad; por tanto, los datos no siguen una distribución normal. Por lo cual, la prueba de hipótesis usará el estadístico de prueba no paramétrica de la U de Mann Whitney a un nivel de significancia de 0,05 .

\section{Prueba de hipótesis general}

Ha: El programa de capacitación influye significativamente en el conocimiento de la crianza respetuosa en la nueva normalidad.

Ho: El programa de capacitación no influye significativamente en el conocimiento de la crianza respetuosa en la nueva normalidad.

Valores:

Nivel mayor $\mathrm{p}>0.05=(\mathrm{Ho})$

Nivel menor $\mathrm{p}<0.05=(\mathrm{Hi})$

Tabla 3

Prueba de hipótesis general

Estadísticos de prueba ${ }^{a}$

\begin{tabular}{lrr}
\hline & $\begin{array}{c}\text { Crianza respetuosa } \\
\text { pretest }\end{array}$ & $\begin{array}{c}\text { Crianza respetuosa } \\
\text { postest }\end{array}$ \\
\hline U de Mann-Whitney & 199,500 &, 000 \\
W de Wilcoxon & 409,500 & 210,000 \\
Z &,- 014 & $-5,427$ \\
Sig. asintótica(bilateral) &, 989 &, 000 \\
\hline
\end{tabular}

a. Variable de agrupación: Grupo

Nota: Base de datos.

Decisión estadística:

Como el valor de significancia observada (sig) $\mathrm{p}=0,000$ es menor al valor de la significancia teórica $\alpha=0,05$ en el postest, nos permite señalar que la diferencia entre el grupo control y el grupo experimental es estadísticamente significativa, por lo tanto, se rechaza la hipótesis nula y se acepta la hipótesis alterna. 


\section{Conclusiones}

El programa de capacitación influye significativamente en el conocimiento de la crianza respetuosa en directivos de instituciones educativas de educación inicial en la nueva normalidad. Este enunciado es confirmado por la prueba estadística U de Mann Whitney que con un $\mathrm{p}=0.000$ $<0.05$ acepta la hipótesis planteada en esta investigación. González y Sáenz (2020) y Di Stefano (2020) consideraron que las propuestas basadas en la crianza respetuosa están vinculadas a establecer patrones orientados al afecto y valores, los cuales permiten que se favorezca el desarrollo humano desde edades tempranas; asimismo, sus resultados coinciden con los de esta investigación pues si se trabajan propuestas basadas en la crianza respetuosa se podrá adquirir progresivamente la autorregulación de emociones para la futura inserción social positiva en niños y niñas.

En cuanto a la dimensión identificar y mantener los objetivos y metas, el programa de capacitación influye significativamente en los directivos de instituciones educativas de educación inicial en la nueva normalidad, sin embargo, se observa un mínimo porcentaje en el nivel de inicio de $5 \%$ que es equivalente a 1 directivo y en el nivel proceso un $10 \%$ equivalente a 2 directivos. Durrant (2013) establece en su publicación que es relevante identificar los objetivos a largo plazo; si deseamos comprender cual es la clave de la disciplina efectiva es importante identificar los logros tratando de sensibilizar a los directores por la búsqueda de soluciones no inmediatas sino consistentes en el tiempo; por ello, los desafíos a corto plazo deben identificarse como oportunidades para trabajar a largo plazo.

Sobre la dimensión proporcionar calidez afectiva y soporte, el programa de capacitación influye significativamente en los directivos de instituciones educativas de educación inicial en la nueva normalidad, sin embargo, se observa un mínimo porcentaje en el nivel de proceso de $15 \%$ que es equivalente a 3 directivos. Tagle (2017) estableció que un liderazgo en instituciones educativas de preescolar debería estar encaminado hacia la organización, transmisión, orientación, y motivación para garantizar el logro de los objetivos previstos, de esta forma al igual que en el presente estudio, el programa fomenta un liderazgo con movilidad y organización, en medida de la comunicación, interacción y el deseo de acceso a nuevo conocimiento.

Referente a la dimensión comprender a la niñez desde sus pensamientos y sentimientos el programa de capacitación influye significativamente en los directivos de instituciones educativas de educación inicial en la nueva normalidad. Mamauag et al. (2021) contribuyeron de manera eficaz con su programa Mapa puesto que desde la mirada de la crianza respetuosa, establecieron al dialogo, como un medio viable para lograr una reducción importante del maltrato infantil y mejorar el bienestar en las familias filipinas, demostrándose de esta forma que en ambas investigaciones, el abordar programas desde un enfoque de fortalecimiento de valores y respeto se podría garantizar cambios significativos para el desarrollo conductual de los niños y niñas.

Y finalmente, sobre la dimensión concentrarse en la solución de los problemas el programa de capacitación influye en los directivos de instituciones educativas de educación inicial en la nueva normalidad, sin embargo, es una de las dimensiones que evidencia menor porcentaje de logros, por ello sería relevante abordarla de manera individual en posteriores investigaciones tal y como lo menciona Anderson (2010) debido a que considera aún muy complejo el identificar las 
Propuesta de capacitación a directores de instituciones educativas del nivel preescolar basado en la crianza respetuosa en la nueva normalidad

características de un tipo de líder en las instituciones educativas, estas se irán evidenciando de diversas formas, pues esto dependerá de los contextos que deberán afrontar y los desafíos que emerjan.

\section{Recomendaciones}

A los directivos de las instituciones educativas de nivel inicial, entender que el capacitarse en temas relacionados a la gestión educativa permite mantener la practica en cuanto a la ejecución de estrategias en virtud del desarrollo de sus instituciones. Pero, el abordar temas de necesidad del contexto social educativo, es aún más necesario, pues es aquí donde se observa el liderazgo para poder encaminar proyectos que permitan brindar una alternativa de solución en esta nueva normalidad.

A los docentes, afianzar aún más esos lazos entre la parte directiva y los padres de familia, puesto que son los actores apropiados para canalizar los requerimientos que tiene la comunidad educativa, al ser los que se interrelacionan constantemente con sus estudiantes y con los padres de familia conociendo sus requerimientos y necesidades en este nuevo contexto.

A los padres de familia, involucrarse activamente con los miembros de sus instituciones educativas para conocer enfoques como el de la crianza respetuosa, los cuales les permitan contar con alternativas referente al cuidado y desarrollo de los niños y niñas en el hogar, en nuevos contextos como los derivados de la pandemia.

\section{Referencias Bibliográficas}

Anderson, S. (2010). Liderazgo directivo: claves para una mejor escuela. Psicoperspectivas, 9(2), 34-52. https://scielo.conicyt.cl/scielo.php?pid=S0718$\underline{69242010000200003 \& \text { script }=\text { sci_arttext }}$

Angulo, S., Prieto, N. y Silva, M. (2021). Diseño y validación de una cartilla sobre crianza con respeto para cuidadores [tesis de pregrado, Pontificia Universidad Javeriana]. Repositorio Institucional $\quad-\quad$ Pontificia Universidad Javeriana. https://repository.javeriana.edu.co/handle/10554/54331

Bustamante, R. (2020). Educación en cuarentena: cuando la emergencia se vuelve permanente (segunda parte). Aportes para el Diálogo y la Acción http://www.grade.org.pe/creer/archivos/articulo-5.pdf

Cable News Network (CNN). (23 de diciembre de 2020). De la educación presencial a la virtual: los desafíos para los estudiantes debido a la pandemia. https://cnnespanol.cnn.com/2020/12/23/de-la-educacion-presencial-a-la-virtual-losdesafios-para-los-estudiantes-debido-a-la-pandemia/

Chiquito, L. (2019). El liderazgo pedagógico en directoras de educación preescolar de la región oriente de la ciudad de Puebla. [Tesis de maestría, Universidad Iberoamericana Puebla]. https://repositorio.iberopuebla.mx/bitstream/handle/20.500.11777/4219/Tesis.pdf?sequen $\underline{\mathrm{ce}=1 \& \text { isAllowed }=\mathrm{y}}$

Di Stefano, D. L. (2020). Crianza con apego: nuevas recetas para viejas prescripciones. Actas de Periodismo 
http://sedici.unlp.edu.ar/bitstream/handle/10915/113500/Documento_completo.pdfPDFA.pdf?sequence $=1 \&$ isAllowed $=\mathrm{y}$

Díaz, M. y Elizalde, B. (2021). Grupos de crianza comunitaria: ¿iniciativas comunitarias o grupos identitarios?. RES. Revista Española de Sociología, 30(2), 6. https://dialnet.unirioja.es/servlet/articulo?codigo=7891661

Duarte, D. (2019). Intervención psicoterapéutica en un caso de problemas de relación entre padres e hijos [tesis de pregrado, Universidad Cooperativa de Colombia]. Repositorio Institucional UCC. https://repository.ucc.edu.co/handle/20.500.12494/15319

Durrant, J. (2013). Disciplina positiva en la crianza cotidiana. https://resourcecentre.savethechildren.net/node/7509/pdf/positive_discipline_in_everyday _parenting_sp_hi-res_final_2017.pdf

Galiya, A., Malika, K., Kuanyshbek, A., Salima, S., y Gulmira, B. (2018). Managerial competence of future specialists of the education system (Preschool education and upbringing) and medicine in the comparative aspect. Opción, 34(85), 44-62. https://www.redalyc.org/articulo.oa?id=31055914002

González, M. y Sáenz, N. (2020). Crianza Respetuosa: Hacia una parentalidad centrada en las niñas $\quad \mathrm{y} \quad$ niños. https://revistas.ucr.ac.cr/index.php/estudios/article/view/44887

Guevara, C. Y., Rugerio, J. P., Hermosillo, A. M. y Corona, L. A. (2020). Aprendizaje socioemocional en preescolar: fundamentos, revisión de investigaciones y propuestas. Revista Electrónica de Investigación Educativa, 22(e26), 1-14. https://doi.org/10.24320/redie.2020.22.e26.2897

Hernández, R., Fernández, C. y Baptista, P. (2014). Metodología de la investigación. Sexta edición. https://bit.ly/3GdWiM9

Instituto Colombiano de Bienestar Familiar. (2015). Crianza Positiva. https://bit.ly/3zPy0FV

Mamauag, B. L., Alampay, L. P., Lachman, J. M., Madrid, B. J., Hutchings, J., Ward, C. L., y Gardner, F. (2021). A south-to-south cultural adaptation of an evidence-based parenting program for families in the philippines. Family Process, 60(4), 1202-1216. https://www.scopus.com/record/display.uri?eid=2-s2.0$\underline{85099111385 \& \text { origin }=\text { resultslist\&zone }=\text { contextBox }}$

Mantilla, M. (2019). Cuerpos, niñez y crianza: cartografías corporales de la infancia en el modelo de crianza respetuosa en Argentina. Revista Uruguaya de Antropología y Etnografía, 4(1), 36-50. $\quad$ http://www.scielo.edu.uy/scielo.php?script=sci_arttext\&pid=S2393$\underline{68862019000100036}$

Marquez, V., Reyes, R., Merchán, M. y Valle, D. (2021). Competencias parentales en el desarrollo socioafectivo en niñas y niños de 3 a 5 años. RECIAMUC, 5(3), 182-195. https://reciamuc.com/index.php/RECIAMUC/article/view/684

Mena Lozada, D. (2017). El rol del director en la gestión de conflictos en el Marco de Buen Desempeño del Directivo. Estudio aplicado a los directores de las instituciones educativas del ámbito de la UGEL La Unión-Piura. [Tesis de maestría, Universidad de Piura, Perú]. https://pirhua.udep.edu.pe/bitstream/handle/11042/3018/MAE_EDUC_354.pdf?sequence

Ministerio de Educación del Perú (2021). Guía de disciplina positiva para el desarrollo de habilidades socioemocionales en la escuela y trabajo con las familias. Repositorio Minedu. https://bit.ly/37QFtHx

Morales Saavedra, E. (2016). Influencia del liderazgo directivo en el clima organizacional de dos centros educativos pertenecientes a una cadena de jardines infantiles y salas cunas de la 
Propuesta de capacitación a directores de instituciones educativas del nivel preescolar basado en la crianza respetuosa en la nueva normalidad

Región Metropolitana. [Tesis de maestría, Pontificia Universidad Católica De Chile]. https://repositorio.uc.cl/handle/11534/21474

Nativ, A., Raschkovan, I. y Schulz, N. (2020). Introducción: ¿Por qué cuestionarlo todo?, No tan terribles - Limites y autonomía en la primera infancia. Una mirada desde la crianza $\begin{array}{llllll}\text { respetuosa } & \text { (pp. } & 17 & - & 25) . & \text { Planeta. }\end{array}$ https://pladlivrosbr0.cdnstatics.com/libros_contenido_extra/44/43366_TPCW_No\%20tan \%20terribles.pdf

Navarro, J., Pérez, F., y Arteaga, M. (2017). Vínculos tempranos: Transformaciones al inicio de la vida. Ediciones Universidad Alberto Hurtado. https://repositorio.uahurtado.cl/bitstream/handle/11242/23894/V\%c3\%adnculostempranos.pdf?sequence $=1 \&$ is Allowed $=\mathrm{y}$

Paliwoda, A. (2020). Acerca de los límites en la primera infancia. [Tesis de grado, Universidad de la República de Uruguay]. https://www.colibri.udelar.edu.uy/jspui/bitstream/20.500.12008/27477/1/tfg_ana_paliwo da_-2020_0.pdf

Raschkovan, I. (2016). Psicoanálisis, prevención y crianza. VIII Congreso Internacional de Investigación y Práctica Profesional en Psicología XXIII Jornadas de Investigación XII Encuentro de Investigadores en Psicología del MERCOSUR. https://www.aacademica.org/000-044/281.pdf

Resolución Viceministerial N097-2020-MINEDU - Ministerio de Educación del Perú (2020). "Disposiciones para el trabajo remoto de los profesores que asegure el desarrollo del servicio educativo no presencial de las instituciones y programas educativos públicos, frente al brote del COVID-19". https://cdn.www.gob.pe/uploads/document/file/729925/RVM_N_097-2020MINEDU.pdf

Romo, C. (2020). La educación socioformativa desde la crisis del COVID-19. https://www.researchgate.net/publication/342991902_La_educacion_socioformativa_des de_la_crisis_del_COVID_19

Rossi Valverde, R., y Rossi Ortiz, R. (2021). Liderazgo directivo, modelo de medida del constructo para aplicación en Educación Básica regular. Revista Andina de Educación, 4(1), 11-22. https://repositorio.uasb.edu.ec/handle/10644/7954

Rueda, C. y Velásquez, Á. (2020). Incidencia del liderazgo directivo en la consolidación del ethos docente y su influencia en la cultura Institucional en Aspaen Preescolar y Maternal Tayana y Aspaen Preescolar Urapanes. [Tesis de maestría, Universidad de la Sabana, Colombia]. https://bit.ly/3zEvGBD

Silva, B., Morales, N., Echevarria, H. y Arvizu, P. (2021). Características del liderazgo transformacional de los directores de educación preescolar según los docentes expertos. Revista Relep-Educación y Pedagogía en Latinoamérica, 3(1), 47-67. https://iquatroeditores.com/revista/index.php/relep/article/view/190

Tagle, F. (2017). Significados que se construyen en relación al liderazgo directivo en preescolar. Congreso nacional de investigación educativa - COMIE. http://comie.org.mx/congreso/memoriaelectronica/v14/doc/2780.pdf

Torre, E., y Rodríguez, M. (2019). Paternidades a distancia: Malestares de padres separados de sus hijas e hijos tras la deportación. Estudios fronterizos, 20. https://doi.org/10.21670/ref.1902023 
Unicef Bolivia. (31 de mayo de 2020). Mamás y papás deben apoyar el aprendizaje de las y los adolescentes en el hogar. https://uni.cf/3Ge3urx

Unicef Perú. (16 de junio de 2021). La mitad de los padres, madres o cuidadores en el Perú sufre de ansiedad, depresión o estrés debido a la pandemia. https://www.unicef.org/peru/comunicados-prensa/mitad-padres-madres-cuidadores-perusufre-ansiedad-estres-depresion-pandemia-covid19

Vallejo, M., y Torres, A. (2020). Concepciones docentes sobre la calidad de los procesos de enseñanza y aprendizaje de la educación preescolar. Revista Electrónica Educare, 24(3), 274-293. https://bit.ly/3q8hwFw

Vargas, L. (2017). Formación de profesionales y padres de familia hacia una crianza consciente, amorosa, respetuosa y humanizada. [Tesis de grado, Universidad Piloto De Colombia]. http://repository.unipiloto.edu.co/handle/20.500.12277/6444 